\title{
Denoising of natural images through robust wavelet thresholding and genetic programming
}

\begin{abstract}
Digital images play an essential role in analysis tasks that can be applied in various knowledge domains, including medicine, meteorology, geology, and biology. Such images can be degraded by noise during the process of acquisition, transmission, storage, or compression. The use of local filters in image restoration may generate artifacts when these filters are not well adapted to the image content as a result of the heuristic optimization of local filters. Denoising methods based on learning procedure are more capable than parametric filters for addressing the conflicts between noise suppression and artifact reduction. In this study, we present a nonlinear filtering method based on a two-step switching scheme to remove both salt-and-pepper and additive white Gaussian noises. In the switching scheme, two cascaded detectors are used to detect noise, and two corresponding estimators are employed to effectively and efficiently filter the noise in an image. In the process of training, a method according to patch clustering is utilized, and genetic programming (GP) is subsequently applied to determine the optimum filter (wavelet-domain filter) for each individual cluster, while in testing part, the optimum filter trained beforehand by GP is recovered and used on the inputted corrupted patch. This adaptive structure is employed to cope with several noise types. Experimental and comparative analysis results show that the denoising performance of the proposed method is superior to that of existing denoising methods as per both quantitative and qualitative assessments.
\end{abstract}

Keyword: Gaussian noise; Genetic programming; Image denoising; Nonlinear filters; Saltand-pepper noise; Switching scheme 